Ovidius explanatus. Traduire et commenter les "Métamorphoses" au Moyen Âge, dir. S. BIANCARDI, P. DELEVILLE, F. MONTORSI, M. POSSAMAÏ-PÉREZ

\title{
Piero Andrea Martina
}

\section{OpenEdition}

\section{Journals}

\section{Edizione digitale}

URL: https://journals.openedition.org/studifrancesi/22227

DOI: 10.4000/studifrancesi.22227

ISSN: 2427-5856

\section{Editore}

Rosenberg \& Sellier

\section{Edizione cartacea}

Data di pubblicazione: 1 avril 2020

Paginazione: 146-147

ISSN: 0039-2944

\section{Notizia bibliografica digitale}

Piero Andrea Martina, "Ovidius explanatus. Traduire et commenter les "Métamorphoses" au Moyen Âge, dir. S. biancardi, P. deleville, F. montorsi, M. possamaï-Pérez», Studi Francesi [Online], 190 (LXIV | I) | 2020, online dal 01 avril 2020, consultato il 03 août 2021. URL: http://journals.openedition.org/studifrancesi/ 22227 ; DOI: https://doi.org/10.4000/studifrancesi.22227

Questo documento è stato generato automaticamente il 3 août 2021.

\section{(c) (i) (2) $\Theta$}

Studi Francesi è distribuita con Licenza Creative Commons Attribuzione - Non commerciale - Non opere derivate 4.0 Internazionale. 


\title{
Ovidius explanatus. Traduire et commenter les "Métamorphoses" au Moyen Âge, dir. S. BIANCARDI, P. DELEVILLE, F. MONTORSI, M. POSSAMAÏ- PÉREZ
}

\author{
Piero Andrea Martina
}

\section{NOTIZIA}

Ovidius explanatus. Traduire et commenter les "Métamorphoses" au Moyen Âge, dir. S. BIANCARDI, P. DELEVILLE, F. MONTORSI, M. POSSAMAï-PÉREZ, Paris, Classiques Garnier, 2018, «Rencontres» 323, $323 \mathrm{pp}$.

1 Il volume, pubblicato nella collezione «Rencontres» a inaugurare la serie Ovidiana, riunisce i contributi presentati in un incontro svoltosi a Lione tra alcuni membri del progetto Ovide en français. Sotto la guida di M. POSSAMAï-PÉREZ, che firma l'introduzione (pp. 7-20) e la postfazione (pp. 287-291, la quale riporta delle considerazioni, presentate nella tavola rotonda conclusiva del colloquio, di Francine Mora e Jacqueline Cerquiglini-Toulet), il volume presenta una forte unità tematica e si impone, in anni di rinnovato interesse per l'Ovide moralisé (OM), anche come strumento d'appoggio all'edizione di questo testo di cui si rende conto in questa stessa rassegna.

2 I contributi sono disposti seguendo un ordine logico-cronologico, dalle fonti al testo e alla sua posterità, con alcuni casi di dialogo proficuo tra gli articoli.

3 Frank Coulson (The Allegories in the Vulgate Commentary, pp. 23-38) si sofferma sul commento cosiddetto Vulgato, di cui l'A. prepara un'edizione. Egli si concentra sulle allegorie che i commentatori medievali giustappongono ai miti ovidiani: quelle del 
Vulgato, commento di provenienza orleanese (terzo quarto del XIII sec. ca) dipendono per buona parte dal commento di Arnolfo d'Orléans, ma non senza alcune interpretazioni originali o la ripresa originale di allegorie già presenti nella tradizione esegetica antecedente.

Con Laura ENDRESS (Un répertoire du type «de montibus et fluminibus» dans l'“Ovide moralisé"? À propos d'un passage interpolé et ses sources possibles, pp. 39-65) entriamo nel vivo degli studi sul testo volgare. L'A. studia un passaggio del mito di Fetonte in cui l'autore dell'OM inserisce una serie di toponimi che estendono e completano la descrizione ovidiana (II, 381-422, 448-512 del testo francese). Parte delle aggiunte può derivare dalla lettura di altri passi ovidiani (delle Metamorfosi, delle Ex Ponto, dei Fasti), o dalle glosse al passo in questione. L'importanza non è tanto ricercare la fonte precisa, ma mettere il testo dell'OM in relazione con la costellazione di testi mitografici e di erudizione classica circolanti tra fine del XIII e inizio del XIV secolo, e che costituiscono un sottobosco in cui si prepara uno sviluppo pre-umanistico che pare essere in continuità con simili testi.

Jean-Yves TILLIETTE (De la prose au vers. L'"Ovide moralisé" traducteur de Hugues de Fouilloy, pp. 67-97) ritorna su un passaggio del XV libro dell'OM da lui già illustrato in precedenza (negli Studi in onore di A. Paravicini Bagliani, Firenze, 2008): nella moralizzazione del discorso di Pitagora (3312-5767), l'autore del testo francese aggiunge un lungo inserto che altro non è se non la traduzione del De medicina animae di Ugo di Fouilloy. Autore, quest'ultimo, cui quello dell'OM doveva sentirsi piuttosto vicino, soprattutto per la volontà di sezionare, in traslato, il mondo - e l'uomo - al fine esegetico. Ma J.-Y. T. si sofferma soprattutto sulle modalità di traduzione, globalmente fedele, del testo, in parte divergenti da quelle del latino delle Metamorfosi anche per il fatto di dover trasporre in versi un testo originariamente in prosa. La traduzione è operazione culturale piena soprattutto nel trasporre la spiritualità cistercense di Ugo in un contesto di buone pratiche devozionali, evidentemente più vicino al pubblico dell'OM.

6 L'inserimento dell'OM in un contesto latino è studiato da Jean-Baptiste GUILLAUMIN (Cosmologie et représentations du monde dans les gloses latines du premier livre de l'"Ovide moralisé", pp. 99-138), che affronta le glosse latine che in alcuni testimoni (tra cui A1, ms. di base già dell'edizione De Boer) accompagnano il testo francese - tema su cui M.R. Jung per primo richiamava l'attenzione almeno dal 1997. L'A. studia in particolare la relazione tra il testo dell'OM e quello delle glosse nel libro I, incentrando la propria analisi sulle glosse cosmologiche (natura del mondo, dell'uomo, di Dio) che dialogano con la cristianizzazione delle Metamorfosi del testo francese.

7 Con l'articolo di David F. HULt ("Ovide moralisé": anonymat et autorité, pp. 141-153) si passa all'analisi del testo dell'OM e in particolare della figura dell'autore, studiata attraverso le considerazioni su Dio.

8 Sulla 'voce narrativa' dell'autore dell'OM insiste pure Francesco MONTORSI (La traduction de la voix narrative dans l'“ovide moralisé", pp. 155-183). Lo studio della sorte delle apostrofi ai personaggi da parte di Ovidio, cancellate nel passaggio al testo francese, porta l'A. ad alcune riflessioni dalla portata più ampia sull'instance narratoriale nell'OM. I risultati più interessanti si trovano nell'analisi delle 'incertezze' narrative di Ovidio: laddove l'autore latino si mostra ironicamente dubbioso nei riguardi dei miti, e delle varianti spesso contrastanti che doveva trovare nelle proprie fonti, l'OM tace. Una volta 
entrati nella 'moralizzazione' del testo, il mito acquisisce uno statuto di verità che non aveva neppure per l'autore (e per il lettore) classico.

9 Ad analoghe conclusioni giunge pure Jean-Marie FRITZ, Moraliser le cannibalisme (Lycaon, Pélops, Térée): les solutions des "Ovides moralisés latins» et français (pp. 219-234) attraverso lo studio dei miti cannibalici presenti nel testo ovidiano e delle soluzioni adottate nell'OM francese e nei testi latini di Arnolfo d'Orléans, Giovanni di Garlandia, Giovanni del Virgilio e Pierre Bersuire. Se ne ricava un processo culturale abbastanza coerente, per cui i miti cannibalici (e incestuosi) sono edulcorati: l'A. propone che ciò sia da vedere come il riflesso del dibattito sulla presenza reale del Cristo nell'eucarestia, che può spiegare la reticenza medievale di fonte a tali miti.

Simone BIANCARDI (Le livre XII de l'“Ovide moralisé": réécritures, entre continuité et discontinuité, pp. 185-201) presenta a volo d'uccello il XII libro dell'OM (di cui prepara l'edizione), insistendo sui passaggi tratti dalle Eroidi e dall'Iliade latina.

11 Da ultimo si passa allo studio della posterità dell'OM, che integra il capitolo sulle mises en prose nell'edizione del testo (ad opera di S. Cerrito). Prunelle DELEVILLE (Une réécriture de l'“Ovide moralisé": les copies de la famille Z, pp. 205-217) affronta questo tema studiando la famiglia $Z$, la cui particolarità è quella di «de-moralizzare» il testo dell'OM. L'A. mostra come per questi quattro manoscritti l'operazione non consista nella semplice soppressione di alcune allegorie, ma in una riscrittura coerente dal punto di vista delle finalità estetiche ed etiche, che parte dalla constatazione dell'avere a che fare con un pubblico diverso. Irene SALVO GARCíA, Les "Métamorphoses" et l'histoire ancienne en France et en Espagne (XIII$\mathrm{XIV}^{\mathrm{e}}$ s.): l'exemple des légendes crétoises (Mét. VII-VIII) (pp. 235-258) ci porta dentro un altro testo, la General estoria «di Alfonso X», in qualche modo parallelo all'OM (e all'Histoire ancienne): tali vicinanze non vanno interpretate nel senso di una dipendenza di un testo dall'altro, ma in quanto sviluppo coerente a partire da uno stesso sistema di fonti, come si evince dallo studio dei miti cretesi dei libri VII e VIII delle Metamorfosi.

Inès HANSEN, De l"“'Ovide moralisé” à l'Ovide figuré: observations sur les premiers imprimés des "Métamorphoses" françaises (pp. 259-285), infine, studia le Metamorfosi ovidiane in francese nelle prime stampe, dalla fine del XV sec. al 1557, così da mostrare in negativo, attraverso la specificità di queste traduzioni che non dipendono più, in generale, dall'OM, alcuni tratti caratteristici di questo testo.

Chiudono il volume un'utile bibliografia e due indici, dei personaggi mitologici e biblici e degli autori ed editori antichi.

L'OM che emerge da questa raccolta di articoli, estremamente coerente, è un testo che si inserisce a pieno titolo nella propria temperie culturale, ma che la trapassa, nella successione delle sue modifiche. Emerge un vero e proprio sistema di testi, in cui lo stesso concetto di fonte è difficile da applicare: soltanto non prescindendo da esso l'OM può essere compreso ed apprezzato appieno. 